\title{
Effects of intracranial meningioma location, size, and surgery on neurocognitive functions: a 3-year prospective study
}

\author{
Evangelia Liouta, MSc, ${ }^{1,2}$ Christos Koutsarnakis, MD, ${ }^{1}$ Faidon Liakos, MD, ${ }^{1}$ and \\ George Stranjalis, MD, $\mathrm{PhD}^{1,2}$
} 'Department of Neurosurgery, National and Kapodistrian University of Athens, Evangelismos Hospital; and ${ }^{2 H e l l e n i c ~ C e n t e r ~ f o r ~}$
Neurosurgical Research "Prof. Petros Kokkalis," Athens, Greece

\begin{abstract}
OBJECTIVE Current recommendations stress the need for cognitive parameters to be integrated in the evaluation of outcomes for intracranial meningioma surgery. The aim of this prospective study was to examine neurocognitive function in meningioma patients pre- and postoperatively.

METHODS Patients with skull base (anterior and middle fossa) and convexity (anterior and posterior) meningiomas ( $\mathrm{n}$ $=54)$ underwent neuropsychological examination prior to and 1 year after surgery. A control group $(n=52)$ of healthy volunteers matched for age, sex, and education underwent the same examination. Assessments included executive, memory, and motor functions with standardized testing. Patients with convexity meningiomas were clinically assessed for parietal association cortex functions.
\end{abstract}

RESULTS All patients performed significantly worse $(p<0.05)$ in most neurocognitive domains than controls. The skull base group showed more disturbances in memory than the convexity group $(p<0.05)$. The anterior convexity group showed more deficits in executive function than the posterior convexity group, which presented with parietal association cortex deficits. Verbal deficits were more pronounced in the left hemisphere than in the right hemisphere. Patients with a large tumor $(>4 \mathrm{~cm})$ had more severe neurocognitive deficits than those with a small tumor $(<4 \mathrm{~cm})$. Postoperatively, patients showed no deterioration in neurocognitive function. Instead, significant improvement $(p<0.05)$ was observed in some executive, motor, and parietal association cortex functions.

CONCLUSIONS According to the authors' findings, intracranial meningiomas may cause neurocognitive deficits in patients. Surgery does not cause a deterioration in cognitive function; instead, it may lead to improvements in some functions. Permanent neuropsychological postoperative deficits should be interpreted as tumor-induced rather than due to surgery.

http://thejns.org/doi/abs/10.3171/2015.6.JNS1549

KEY WORDS skull base meningioma; convexity meningioma; surgery; cognition; prospective study; oncology

$\mathrm{M}$ ENINGIOMAS constitute approximately $15 \%-20 \%$ of all intracranial neoplasms and are often associated with favorable clinical prognosis because of their benign histology. Because of the high survival rates associated with meningiomas, patients with meningiomas can expect increased functional capacity compared with patients harboring other brain tumors. Parameters such as the rate of survival, tumor recurrence, and progres- sion, ${ }^{8}$ as well as the quality of life ${ }^{3,17}$ of these patients, have traditionally been used to assess outcomes after intracranial meningioma surgery. According to recommendations from current outcomes research programs, neuropsychological-cognitive parameters also need to be incorporated to comprehensively evaluate surgical outcomes.

Although meningiomas are extraaxial tumors, cognitive decline may arise due to perilesional edema and mass

ABBREVIATIONS COWAT = Controlled Oral Word Association Test; DWI = diffusion-weighted imaging; RBMT = Rivermead Behavioural Memory Test Extended Version; TMT = Trail Making Test.

SUBMITTED January 9, 2015. ACCEPTED June 17, 2015.

INCLUDE WHEN CITING Published online December 4, 2015; DOI: 10.3171/2015.6.JNS1549. 
effect on normal cerebral tissue. Compared with healthy subjects, meningioma patients have reduced psychomotor speed and working memory ${ }^{27}$ and attentional dysfunctions. ${ }^{14,18}$ Furthermore, an association between neuropsychiatric symptoms and the occurrence of meningiomas, in particular of the anterior fossa, has been observed. ${ }^{1,2,13}$

Regarding postoperative neurocognitive outcomes, the literature suggests that about $40 \%$ of patients experience cognitive or emotional problems following surgery. ${ }^{26} \mathrm{~Pa}-$ tients with meningioma show significant long-term impairments in executive functioning; ${ }^{6}$ those with skull base meningiomas perform lower on cognitive testing than those with convexity meningiomas, and those with leftsided meningiomas show more verbal memory deficits than those with right-sided meningiomas. ${ }^{28}$

In general, neurocognitive deficits have been documented preoperatively in patients with meningiomas, suggesting that the tumor itself can cause neuropsychological disturbances. Furthermore, a number of studies have suggested that patients with surgically treated meningiomas present postoperatively with cognitive declines. However, those studies are retrospective and do not take into account preoperative cognitive status. To investigate whether postoperative cognitive sequelae are due to tumor or surgery, prospective studies need to be conducted. So far, the literature lacks prospective studies with large samples of meningioma patients. The aim of our prospective study was to evaluate tumor and surgery effects on neurocognitive function in patients harboring skull base, anterior convexity, and posterior convexity meningiomas.

\section{Methods}

\section{Participants}

During 2011-2013, 61 patients with intracranial meningiomas were admitted for neurosurgical treatment to the University Neurosurgical Clinic of Evangelismos Hospital. Of these patients, 54 right-handed patients (38 females, mean age $56.8 \pm 12.8$ years $[ \pm \mathrm{SD}]$ ) with intracranial skull base (anterior and middle fossa) and convexity (frontal and parietal) meningiomas, and a control group of 52 healthy volunteers, matched for age, sex, and education, were enrolled in this prospective study. The remaining 7 patients were excluded from the study for the following reasons: 2 patients were receiving antipsychotic agents, 2 patients had en plaque meningioma, 1 patient was foreign and neuropsychological assessment could not be offered in his native language, 1 patient refused to enroll in the study, and 1 patient had recurrent meningioma. The enrolled patients underwent neuropsychological assessment prior to and 1 year following surgery. None of the enrolled patients had a previous history of intracranial mass lesion and/or other neurological diseases. Tumor histology and total tumor resection were confirmed by neuropathological and radiological assessments, respectively. The healthy control group underwent the same neuropsychological assessment to compare their performance with patient preoperative assessments. Consent forms were obtained from all participants prior to testing. The participants' demographic and clinical data are presented in Table 1.
TABLE 1. Participants' demographic and clinical data

\begin{tabular}{|c|c|c|}
\hline Variable & Control Group & Patient Group \\
\hline No. of participants & 52 & 54 \\
\hline Sex (F/M) & $34 / 16$ & $38 / 16$ \\
\hline Mean age (yrs) & $53.2 \pm 10.9$ & $56.8 \pm 12.8$ \\
\hline Handedness (rt/lt) & $51 / 1$ & $54 / 0$ \\
\hline Mean duration of education (yrs) & $11.5 \pm 1.9$ & $10.2 \pm 2.4$ \\
\hline Symptoms & NA & \\
\hline Seizure & & $37.1 \%$ \\
\hline Headache & & $27.1 \%$ \\
\hline Gait disturbances, dizziness & & $12.9 \%$ \\
\hline Visual disturbances & & $9.7 \%$ \\
\hline No symptoms & & $8.1 \%$ \\
\hline Psychiatric symptoms & & $4.8 \%$ \\
\hline Medication* & NA & \\
\hline Anticonvulsants & & $22.3 \%$ \\
\hline Steroids & & $26.6 \%$ \\
\hline Maximal mass diameter (cm) & NA & $4.8 \pm 1.4$ \\
\hline Lesion lateralization & NA & \\
\hline $\mathrm{Lt}$ & & 17 \\
\hline Rt & & 27 \\
\hline Bilat & & 10 \\
\hline Lesion localization & NA & \\
\hline Convexity & & 28 \\
\hline Skull base & & 26 \\
\hline
\end{tabular}

\section{Materials}

Cognitive assessments consisted of a standardized neuropsychological battery of the following tests: Wechsler Adult Intelligence Scale-III Digit Span subtest, ${ }^{29}$ a test of short-term and working memory; Trail Making Test (TMT) Parts A and $\mathrm{B},{ }^{31} \mathrm{a}$ test of speed processing and executive function; Controlled Oral Word Association Test (COWAT) (phonemic and category subsets ${ }^{16}$ ), a test for verbal fluency; and Rivermead Behavioural Memory Test Extended Version (RBMT) story and route immediate and delayed recall subtests, ${ }^{5}$ to test recall of new verbal and nonverbal information. Furthermore, fine motor function of the upper extremities was assessed using the finger-tapping test. ${ }^{20}$ All tests were administered and scored according to standard procedures provided in their respective test manuals. Finally, patients were clinically evaluated for disorders that usually result from parietal association cortex damage such as apraxia, agnosia, and sensory loss.

\section{Statistical Analysis}

Data were analyzed using the SPSS software package (version 21). According to statistical analysis, the data were normally distributed. Thus, independent sample ttests (significance level set at $\mathrm{p} \leq 0.05$ ) were used to compare neuropsychological performance between patient and control groups. Independent t-tests were also used to in- 
vestigate the effects of localization (skull base vs convexity, anterior skull base vs middle skull base, and anterior convexity vs posterior convexity), lateralization (left vs right), and size of the tumor in patients during the preoperative neuropsychological performance assessment. Paired sample t-tests were used in the patient group to evaluate the effect of surgery on neuropsychological functions. Bonferroni corrections were applied for multiple comparisons. Finally, 1-way ANOVA and Tukey's post hoc analysis were used to examine the effect of edema on preoperative neuropsychological performance.

\section{Results}

\section{Preoperative Assessments}

Patients Versus Controls

Statistical comparisons between patients with meningiomas and healthy volunteers revealed statistically significant differences in neuropsychological performance. Overall, the patients had markedly lower scores in the Digit Span Backward subtest, COWAT phonological and semantic subtests, finger-tapping test for hands, TMT Part B, and RBMT delayed story recall (see Table 2). The Digit Span Forward subtest, the TMT Part A, the RBMT immediate story, and the RBMT immediate and delayed route recall were comparable between the patient and control groups.

\section{Right Versus Left Hemisphere}

Patients were divided into 2 groups according to tumor lateralization: the left hemisphere group $(n=17)$ and the right hemisphere group $(n=27)$. Patients with bilateral meningiomas were excluded from the analyses. Statistical comparisons showed that the left hemisphere group scored significantly lower than the right hemisphere group in phonological $(\mathrm{p}=0.018)$ and semantic $(\mathrm{p}=0.001)$ COWAT, and RBMT immediate $(\mathrm{p}=0.021)$ and delayed $(\mathrm{p}=0.016)$ story recall. The remaining variables did not show statistically significant differences between the two groups.

\section{Convexity Versus Skull Base}

Patients were subdivided into 2 groups according to lesion localization: convexity meningioma patients $(\mathrm{n}=28)$ and skull base meningioma patients $(n=26)$. Statistical comparisons revealed that skull base meningioma patients showed poorer performance on the memory tests RBMT immediate story $(\mathrm{p}=0.017)$ and route $(\mathrm{p}=0.028)$ recall and the RBMT delayed story $(\mathrm{p}=0.025)$ and route $(\mathrm{p}=$ $0.029)$ recall when compared with the convexity group.

\section{Anterior Versus Posterior Convexity}

Patients with convexity meningiomas were divided into anterofrontal $(n=16)$ and posteroparietal $(n=12)$ convexity groups. The anterofrontal group scored significantly lower on Digit Span Backward ( $\mathrm{p}=0.022)$, COWAT phonological $(p=0.026)$, and TMT Part B $(p=0.019)$ tests compared with the posteroparietal group. In addition, 7 of 12 patients with parietal meningiomas presented with relevant disturbances: 1 patient (with right superior lobule meningioma) had asomatagnosia, 1 patient (with a left inferior and superior parietal lobules meningioma) had perceptual visual agnosia, ideokinetic apraxia, and left-right disorientation, 1 patient (with a left posterior parietal lobe meningioma) had only ideokinetic apraxia, 1 patient (with a left inferior parietal lobule meningioma) had anomic aphasia and left-right disorientation, 1 patient (with a right parietal lobe meningioma) had perseverations in visual and tactile stimuli, and 2 patients (with right inferior mainly parietal lobule meningiomas) showed visual and tactile double extinction. None of the 16 patients with anterior (frontal) convexity meningiomas presented with the aforementioned deficits.

TABLE 2. Participants' performance on neuropsychological testing*

\begin{tabular}{|c|c|c|c|c|c|}
\hline Variable & Control Group & Patient Group Preop & $\begin{array}{c}\text { Controls/Patients } \\
\text { Comparison }\end{array}$ & Patient Group Postop & $\begin{array}{l}\text { Patient Pre-Post } \\
\text { Comparison }\end{array}$ \\
\hline COWAT phonemic & $30.2 \pm 6.3(45)$ & $19.8 \pm 7.2(6)$ & $<0.001 \dagger$ & $26.4 \pm 8.1(48)$ & $<0.001 \dagger$ \\
\hline COWAT semantic & $48.4 \pm 7.6(47)$ & $34.0 \pm 9.2(4)$ & $<0.001 \dagger$ & $42.7 \pm 12.1(19)$ & $0.001 \dagger$ \\
\hline DS Forward & $8.3 \pm 1.6$ & $7.5 \pm 1.5$ & 0.368 & $7.5 \pm 1.2$ & 0.923 \\
\hline DS Backward & $6.2 \pm 1.3$ & $3.9 \pm 1.6$ & $<0.002 \dagger$ & $4.3 \pm 1.2$ & 0.482 \\
\hline TMT A & $52.7 \pm 19.9(56)$ & $70.6 \pm 27.1(9)$ & 0.033 & $48.7 \pm 22.7(54)$ & $0.002 \dagger$ \\
\hline TMT B & $102.9 \pm 33.4(50)$ & $136.8 \pm 46.2(6)$ & $<0.001 \dagger$ & $128.5 \pm 78.1(12)$ & 0.118 \\
\hline Rt finger tapping & $46.1 \pm 8.1(40)$ & $33.9 \pm 10.0(14)$ & $<0.002 \dagger$ & $42.4 \pm 9.7(48)$ & $0.001 \dagger$ \\
\hline Lt finger tapping & $42.8 \pm 9.6(32)$ & $31.5 \pm 10.5(12)$ & $0.002 \dagger$ & $34.9 \pm 12.0(16)$ & 0.667 \\
\hline RBMT story immediate recall & $9.2 \pm 2.1(44)$ & $5.9 \pm 1.4(2)$ & 0.036 & $6.4 \pm 2.0(3)$ & 0.705 \\
\hline RBMT story delayed recall & $8.5 \pm 1.8(52)$ & $5.8 \pm 1.7(12)$ & $<0.002 \dagger$ & $5.9 \pm 1.4(13)$ & 0.185 \\
\hline RBMT route immediate recall & $13.6 \pm 1.6(59)$ & $12.8 \pm 2.3(46)$ & 0.839 & $13.2 \pm 2.6(53)$ & 0.821 \\
\hline RBMT route delayed recall & $13.0 \pm 1.6(62)$ & $12.9 \pm 1.9(60)$ & 0.612 & $12.6 \pm 3.1(57)$ & 0.649 \\
\hline
\end{tabular}

DS = Digit Span.

* Raw data are presented as the mean \pm SD. Standardized scores are shown in parentheses as percentile scores.

$\dagger p$ values are significant at the 0.05 level adjusted for multiple comparisons with Bonferroni correction. 


\section{Anterior Versus Middle Skull Base Location}

Patients with skull base meningiomas were divided into anterior skull base (anterior cranial fossa, $\mathrm{n}=11$ ) and middle skull base (middle cranial fossa, $\mathrm{n}=14$ ) groups. The anterior skull base group scored significantly higher on the memory RBMT immediate $(\mathrm{p}=0.019)$ and delayed $(\mathrm{p}=0.012)$ story recall as well as on the RBMT delayed route recall $(\mathrm{p}=0.024)$.

\section{Small Versus Large Tumor}

Patients were subdivided into 2 groups according to lesion size: patients in the small meningioma group were characterized as harboring a mass that was smaller than $4 \mathrm{~cm}$ in maximum diameter $(\mathrm{n}=24)$ and those in the large meningioma group were characterized as harboring a mass greater than $4 \mathrm{~cm}$ in maximum diameter $(\mathrm{n}=$ 30). Statistical comparisons between the 2 groups showed that patients with large lesions had poorer performance on language and fine motor testing than patients with small lesions. There was a statistically significant difference in the phonological $(\mathrm{p}=0.021)$ and COWAT, and TMT Part $B(p=0.001)$ performance. To better understand the trend of neuropsychological performance according to tumor size, a histogram of the 54 tumor sizes (Fig. 1), as well as the average of preoperative neuropsychological scores by quartiles of tumor size (Fig. 2), is provided for functions that were deficient when compared with control croup.

\section{Edema Versus No Edema}

Patients were divided into those with no tumor edema $(n=17)$, those with peritumoral edema $(n=21)$, and those with extensive widespread edema $(n=16)$. Post hoc analyses on frontal convexity group showed that patients with extensive edema $(n=7)$ had poorer performance on phonological $(p=0.021)$ verbal fluency when compared with patients with no edema $(n=4)$ but not when compared with those with peritumoral edema $(n=5)$. Along the same lines, all but one patient with a posterior convexity meningioma and parietal association deficits had edema (2 peritumoral and 4 extensive) and the majority of patients (4 of 5) with no deficits had no edema. There was no statistical difference in cognition among the 3 edema groups for skull base sample.

\section{Postoperative Assessments}

Paired t-test comparisons of the patients' pre- and postoperative performance revealed no overall significant deterioration in cognitive function. Specifically, postoperative performance on the Digit Span Forward and Digit Span Backward subtests, TMT Part B, the left finger tapping, and the RBMT memory subtests did not show statistically significant differences compared with preoperative performance. Instead, marked improvements were found in the COWAT phonological and semantic, TMT Part A, and right finger-tapping test (Table 2). In addition, all the parietal lobe phenomena that were present in the posterior convexity group were absent at the postoperative examination, and none of the patients developed new parietal lobe phenomena.

\section{Discussion}

Prospective studies on pre- and postoperative neurocognitive function in meningioma patients are scarce. In the present study, neuropsychological functioning before and after surgery in patients with convexity and skull base meningiomas was investigated.

At baseline, patients overall presented with deficits in core domains of cognition, as language, memory, attention, visuospatial function, and fine motor performance were found to be significantly lower than those in the control group. This is in agreement with previous studies suggesting that meningiomas can cause neurocognitive and behavioral deficits, ${ }^{25}$ probably due to compression effects on normal brain tissue. ${ }^{4}$

With regard to tumor laterality, patients with left-sided meningiomas had decreased verbal fluency and recall for verbal material compared with patients with right-sided meningiomas. This finding supports the well-established

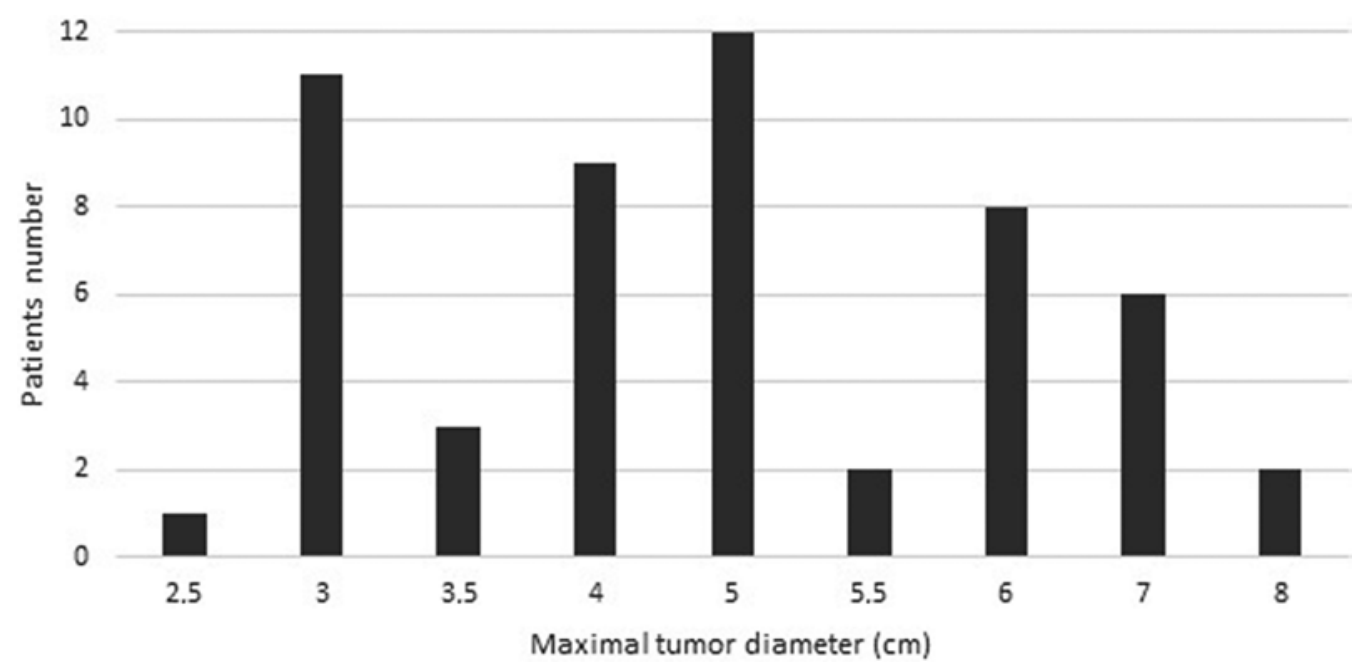

FIG. 1. Histogram showing the frequency of tumor sizes in 54 patients. 


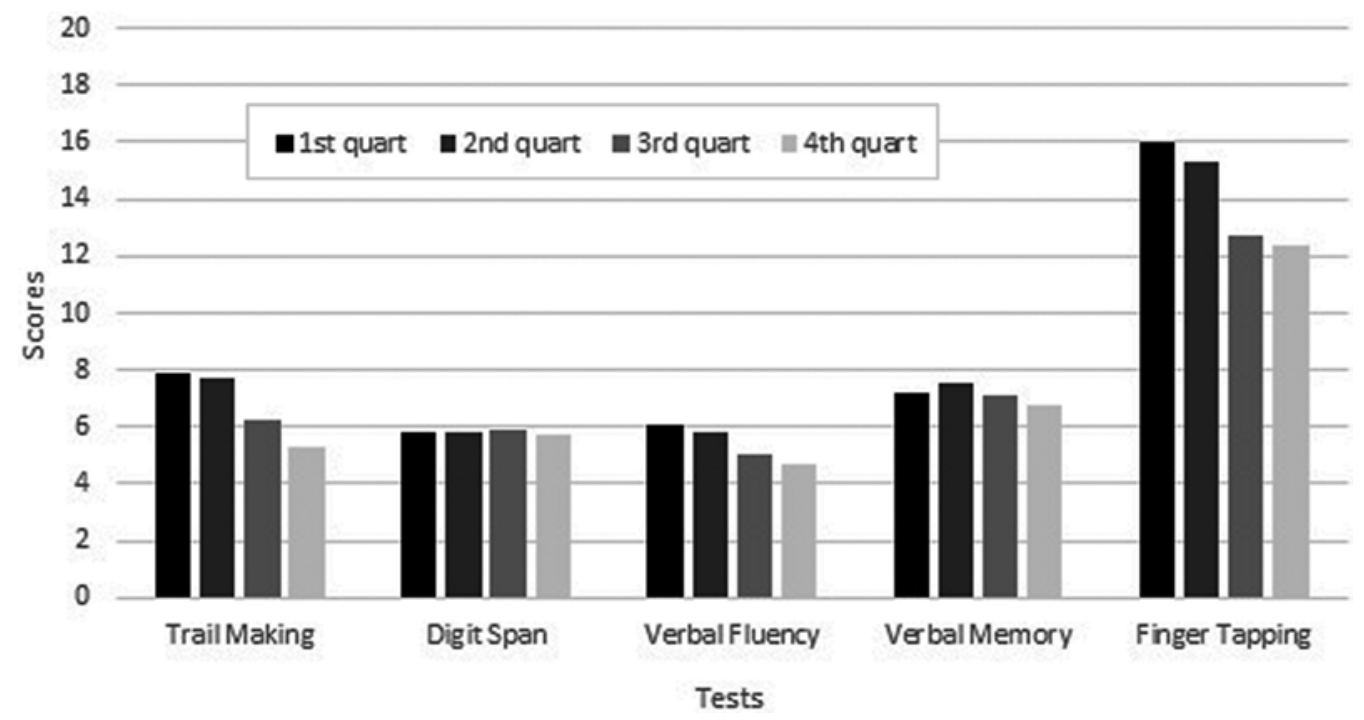

FIG. 2. Average neuropsychological scores by quartile of tumor size. Scores = standard scores; 1 st quartile (quart), $\leq 3.0 \mathrm{~cm}$ (maximum diameter); 2 nd quartile, $\geq 3.5 \mathrm{~cm}$ to $\leq 4.0 \mathrm{~cm}$ (maximum diameter); $3 \mathrm{rd}$ quartile, $\geq 5.0 \mathrm{~cm}$ to $\leq 5.5 \mathrm{~cm}$ (maximum diameter); and 4th quartile, $\geq 6.0 \mathrm{~cm}$ to $\leq 8.0 \mathrm{~cm}$ (maximum diameter).

notion that the left hemisphere is dominant for language function ${ }^{15}$ and is in agreement with previous studies reporting verbal deficits in patients with left-hemisphere benign, ${ }^{28}$ low-grade, ${ }^{9}$ and high-grade malignancy ${ }^{12}$ tumors.

Regarding the superoinferior axis, patients with skull base meningiomas had more deficits in memory components and long-term verbal and nonverbal memory than those with convexity meningiomas. Within the skull base group, patients with middle cranial fossa meningiomas showed greater memory disturbances than those with anterior cranial fossa meningiomas. We considered this finding a result of anatomical proximity between the middle cranial fossa and temporal lobes. Medial temporal structures are well known to support memory function. Previous case reports ${ }^{10,11}$ have shown that skull base mass lesions can affect typical function in the temporal lobe, such as long-term memory.

Within the convexity group, patients with anterior convexity meningiomas had more deficits in executive function than those with posterior convexity meningiomas. The association between frontal lobe lesions and impairment of executive function has been observed previously in a number of studies, ${ }^{7}$ including extraaxial mass lesions. ${ }^{25} \mathrm{On}$ the other hand, posterior convexity meningioma patients presented with parietal association cortex disturbances. Parietal lobe association deficits have been reported in parietal glial tumor patients before and after resection of the parietal lobe. ${ }^{21,22}$ To the best of our knowledge, the current study is the first study to report that extraaxial neoplasms may also cause parietal association cortex deficits.

Neurocognitive function was found to be deficient in meningioma patients in our study, and the pattern of deficiency, as described above, was associated with tumor localization. The severity of deficiency, however, was associated with tumor size. According to our study, neuropsychological deficits were more severe in large meningi- omas than in small ones. Previous studies have shown that the severity of neuropsychological deficits is positively associated with lesion size in glial tumors. ${ }^{12,21}$ Our study shows that this association also exists with meningiomas. These results should be taken into account by neurosurgeons who follow a conservative approach in meningioma treatment. Surgeons should bear in mind that meningioma growth, although typically very slow, may be accompanied by worsening of neuropsychological function.

Regarding postoperative outcomes, the literature suggests the possibility of late neurocognitive sequelae in patients who have undergone surgical treatment for meningiomas. However, currently it is unclear whether these deficits are due to the tumor or are surgically induced. Our comparisons of pre- and postoperative evaluations revealed no overall deterioration in neurocognitive function. Instead, a significant improvement was observed in some of the frontal lobe functions, in verbal fluency, in processing speed (an aspect of visual attention), and in fine movement speed. In addition, all parietal cortex deficits had disappeared by the time of postoperative assessment. On the other hand, short-term memory, working memory, and long-term memory did not show statistically significant improvements postoperatively.

Our postoperative results overall support those in the study by Tucha and colleagues, ${ }^{24,25}$ which showed no cognitive deterioration after surgery in patients with frontal meningiomas, but instead showed that some attention functions were improved. However, our results are in disagreement with studies showing that patients with left-hemisphere meningiomas should not expect any improvement postoperatively. ${ }^{30}$ Our patients exhibited improvement in verbal fluency, a function known to be supported mainly by the left dorsolateral prefrontal cortex..$^{19}$ We considered this disparity probably due to less sensitive assessment tools used in the previous study, as the 
Modified Mini-Mental State Examination is considered a screening instrument for dementia rather than a test to detect subtle cognitive deficits. These results imply that cognitive deficits seen postoperatively in meningioma patients are more likely to be tumor induced rather than surgically induced and present before surgery. Our study stresses the need for a baseline neurocognitive evaluation prior to interpretation of late neurocognitive sequelae in meningioma patients.

In general, neurocognitive function in brain tumor patients can be affected by other factors such as medication or postoperative complications (e.g., stroke). It has been reported that antiepileptic agents may cause cognitive decline, whereas steroids may alleviate cognitive deficits due to resolution of brain edema. ${ }^{23}$ However, during preoperative assessment only a minority of our patients had been receiving antiepileptic agents and steroids; during postoperative examination none of the patients were taking steroids, and only $6 \%$ of patients were receiving antiepileptic agents. Therefore, we consider that the preoperative neurocognitive deficits and any postoperative neurocognitive changes seen in our study were not due to medications, but rather were induced by tumor and surgery, respectively. However, future studies should examine the impact of antiepileptic drugs and steroids on cognition in meningioma patients. Regarding postoperative stroke, one would assume that diffusion weighted imaging (DWI) changes would be correlated with postoperative neurocognitive function. According to our postoperative imaging results none of the patients had DWI changes; therefore, we consider patients' postoperative neurocognitive function not to have been affected by postoperative stroke. However, we suggest that future studies should address this issue and investigate the relationship between postoperative cognition status and DWI results in case of a postoperative stroke.

\section{Conclusions}

Our study shows that typical meningiomas can cause neurocognitive deficits depending on tumor localization and size. Neurosurgery does not result in deterioration in overall neuropsychological function; rather, some function may be improved.

\section{References}

1. Andy OJ, Webster JS, Carranza J: Frontal lobe lesions and behavior. South Med J 74:968-972, 1981

2. Avery TL: Seven cases of frontal tumour with psychiatric presentation. Br J Psychiatry 119:19-23, 1971

3. Chan RC, Thompson GB: Morbidity, mortality, and quality of life following surgery for intracranial meningiomas. A retrospective study in 257 cases. J Neurosurg 60:52-60, 1984

4. Cohen BA, Knopp EA, Rusinek H, Liu S, Gonen O: Brain compression without global neuronal loss in meningiomas: whole-brain proton MR spectroscopy report of 2 cases. AJNR Am J Neuroradiol 26:2178-2182, 2005

5. de Wall C, Wilson BA, Baddeley AD: The Extended Rivermead Behavioural Memory Test: a measure of everyday memory performance in normal adults. Memory 2:149-166, 1994

6. Dijkstra M, van Nieuwenhuizen D, Stalpers LJ, Wumkes M,
Waagemans M, Vandertop WP, et al: Late neurocognitive sequelae in patients with WHO grade I meningioma. J

Neurol Neurosurg Psychiatry 80:910-915, 2009

7. Filley CM: Clinical neurology and executive dysfunction. Semin Speech Lang 21:95-108, 2000

8. Gijtenbeek JM, Hop WC, Braakman R, Avezaat CJ: Surgery for intracranial meningiomas in elderly patients. Clin Neurol Neurosurg 95:291-295, 1993

9. Goldstein B, Obrzut JE, John C, Hunter JV, Armstrong CL: The impact of low-grade brain tumors on verbal fluency performance. J Clin Exp Neuropsychol 26:750-758, 2004

10. Goto H, Nishizaki T, Kajiwara K, Ohmoto Y, Akimura T, Kato S, et al: Improvement in cognitive function after radical excision of an anterior skull base meningioma-a report of 2 cases. J Clin Neurosci 10:375-378, 2003

11. Graziani N, Bouillot P, Dufour H, Donnet A, Ndoye N, Peragut JC, et al: [Meningioma of the floor of the temporal fossa. Anatomo-clinical study of 11 cases.] Neurochirurgie 40:109-115, 1994 (Fr)

12. Habets EJ, Kloet A, Walchenbach R, Vecht CJ, Klein M, Taphoorn MJ: Tumour and surgery effects on cognitive functioning in high-grade glioma patients. Acta Neurochir (Wien) 156:1451-1459, 2014

13. Hunter R, Blackwood W, Bull J: Three cases of frontal meningiomas presenting psychiatrically. BMJ 3:9-16, 1968

14. Jo MY, Armstrong C, Hunter J: Neuropsychological deficits in patients with meningiomas. Arch Clin Neuropsychol 15:718-719, 2000 (Abstract)

15. Kolb B, Whishaw IQ: Fundamentals of Human Neuropsychology. New York: W. H. Freeman, 1990

16. Kosmidis MH, Vlahou CH, Panagiotaki P, Kiosseoglou G: The verbal fluency task in the Greek population: normative data, and clustering and switching strategies. J Int Neuropsychol Soc 10:164-172, 2004

17. Krupp W, Klein C, Koschny R, Holland H, Seifert V, Meixensberger J: Assessment of neuropsychological parameters and quality of life to evaluate outcome in patients with surgically treated supratentorial meningiomas. Neurosurgery 64:40-47, 2009

18. Leimkuhler ME, Mesulam MM: Reversible go-no go deficits in a case of frontal lobe tumor. Ann Neurol 18:617-619, 1985

19. Ravnkilde B, Videbech P, Rosenberg R, Gjedde A, Gade A: Putative tests of frontal lobe function: a PET-study of brain activation during Stroop's Test and verbal fluency. J Clin Exp Neuropsychol 24:534-547, 2002

20. Reitan RM, Wolfson D: The Halstead-Reitan Neuropsychological Test Battery. Tucson, AZ: Neuropsychology Press, 1985

21. Russell SM, Elliott R, Forshaw D, Kelly PJ, Golfinos JG: Resection of parietal lobe gliomas: incidence and evolution of neurological deficits in 28 consecutive patients correlated to the location and morphological characteristics of the tumor. J Neurosurg 103:1010-1017, 2005

22. Sanai N, Martino J, Berger MS: Morbidity profile following aggressive resection of parietal lobe gliomas. J Neurosurg 116:1182-1186, 2012

23. Taphoorn MJB, Klein M: Cognitive deficits in adult patients with brain tumours. Lancet Neurol 3:159-168, 2004

24. Tucha O, Smely C, Lange KW: Effects of surgery on cognitive functioning of elderly patients with intracranial meningioma. Br J Neurosurg 15:184-188, 2001

25. Tucha O, Smely C, Preier M, Becker G, Paul GM, Lange KW: Preoperative and postoperative cognitive functioning in patients with frontal meningiomas. J Neurosurg 98:21-31, 2003

26. van der Vossen S, Schepers VP, Berkelbach van der Sprenkel JW, Visser-Meily JM, Post MW: Cognitive and emotional 
problems in patients after cerebral meningioma surgery. $\mathbf{J}$ Rehabil Med 46:430-437, 2014

27. van Nieuwenhuizen D, Ambachtsheer N, Heimans JJ, Reijneveld JC, Peerdeman SM, Klein M: Neurocognitive functioning and health-related quality of life in patients with radiologically suspected meningiomas. J Neurooncol 113:433-440, 2013

28. Waagemans ML, van Nieuwenhuizen D, Dijkstra M,

Wumkes M, Dirven CM, Leenstra S, et al: Long-term impact of cognitive deficits and epilepsy on quality of life in patients with low-grade meningiomas. Neurosurgery 69:72-79, 2011

29. Wechsler D: Wechsler Adult Intelligence Scale, ed 3. San Antonio: Pearson/The Psychological Corporation, 1997

30. Yoshii Y, Tominaga D, Sugimoto K, Tsuchida Y, Hyodo A, Yonaha $\mathrm{H}$, et al: Cognitive function of patients with brain tumor in pre- and postoperative stage. Surg Neurol 69:5161,2008

31. Zalonis I, Kararizou E, Triantafyllou NI, Kapaki E, Papageorgiou S, Sgouropoulos P, et al: A normative study of the trail making test A and B in Greek adults. Clin Neuropsychol 22:842-850, 2008

\section{Disclosures}

The authors report no conflict of interest concerning the materials or methods used in this study or the findings specified in this paper.

\section{Author Contributions}

Conception and design: Liouta. Acquisition of data: all authors. Analysis and interpretation of data: Liouta. Drafting the article: Liouta. Critically revising the article: Koutsarnakis, Liakos, Stranjalis. Reviewed submitted version of manuscript: all authors. Approved the final version of the manuscript on behalf of all authors: Liouta. Statistical analysis: Liouta. Study supervision: Stranjalis.

\section{Correspondence}

Evangelia Liouta, Department of Neurosurgery, University of Athens, Evangelismos Hospital, Ploutarchou 3 Str., Athens GR 10675, Greece. email: evangelialiouta286@hotmail.com. 